
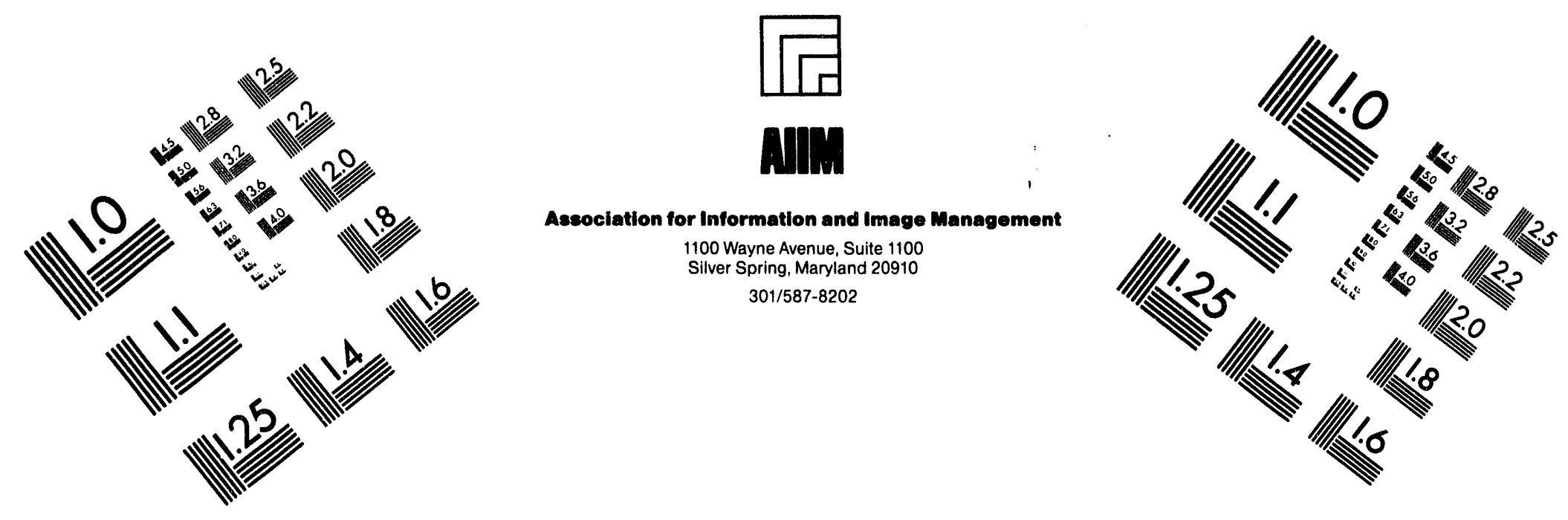

Centimeter

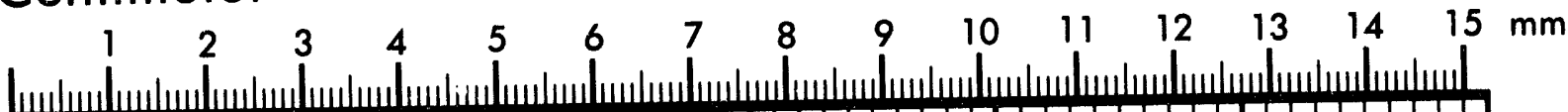
$\mid$ Inches
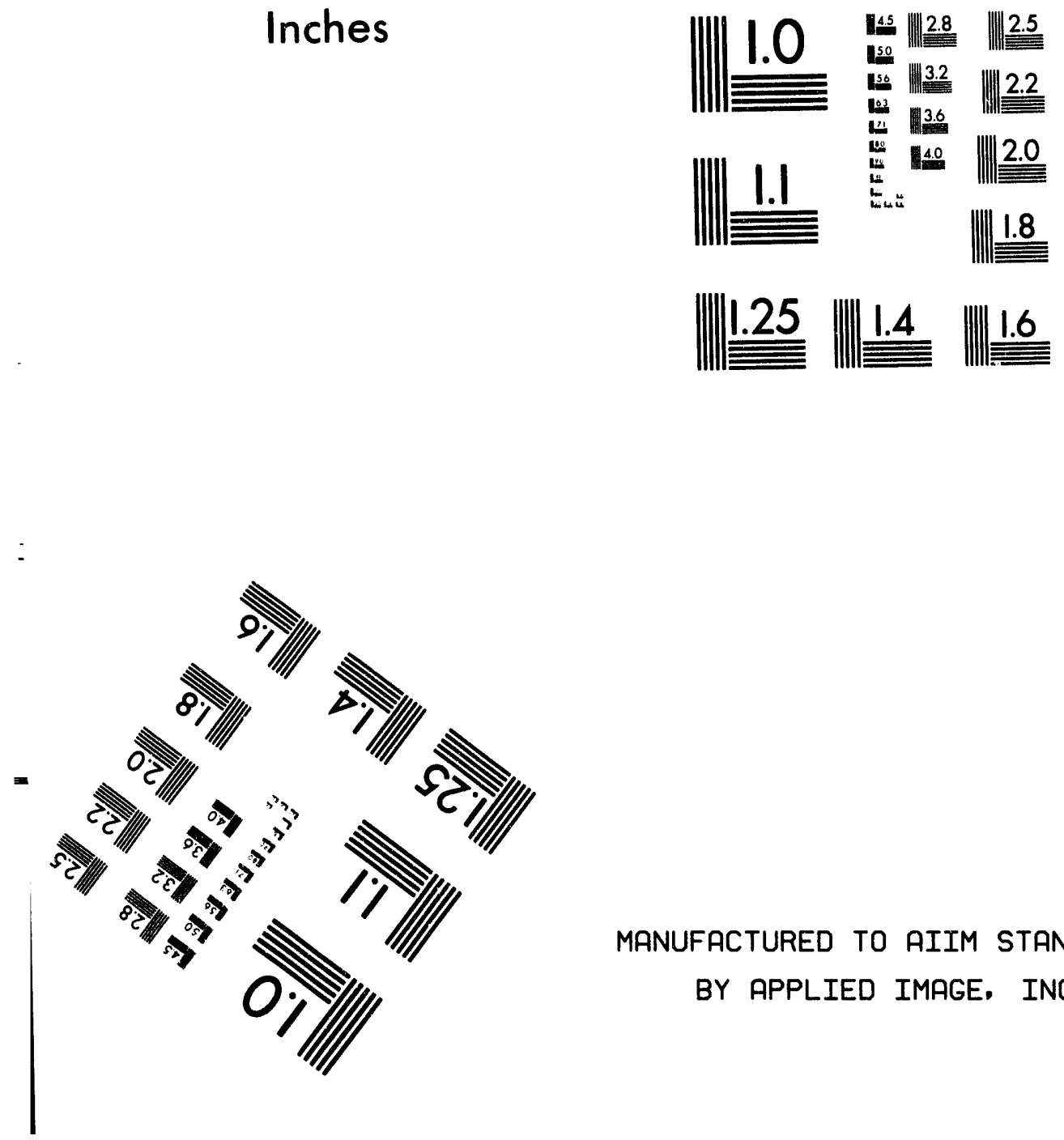

MANUFACTURED TO AIIM STANDARDS BY APPLIED IMAGE, INC.

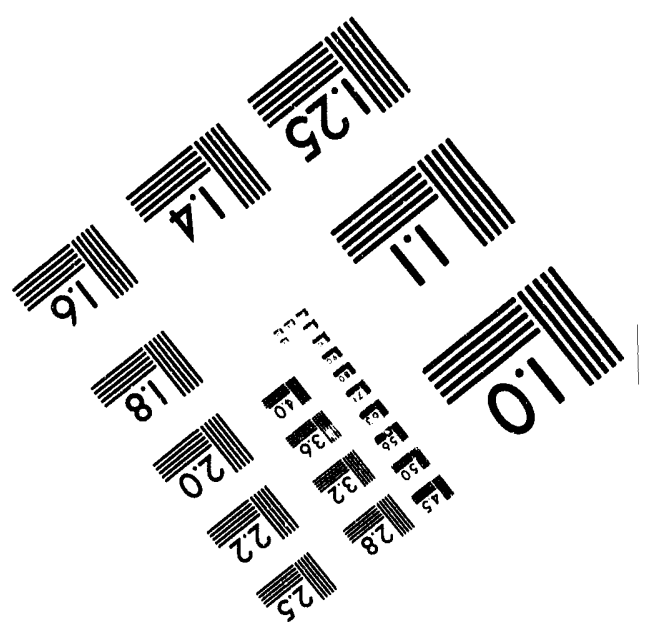



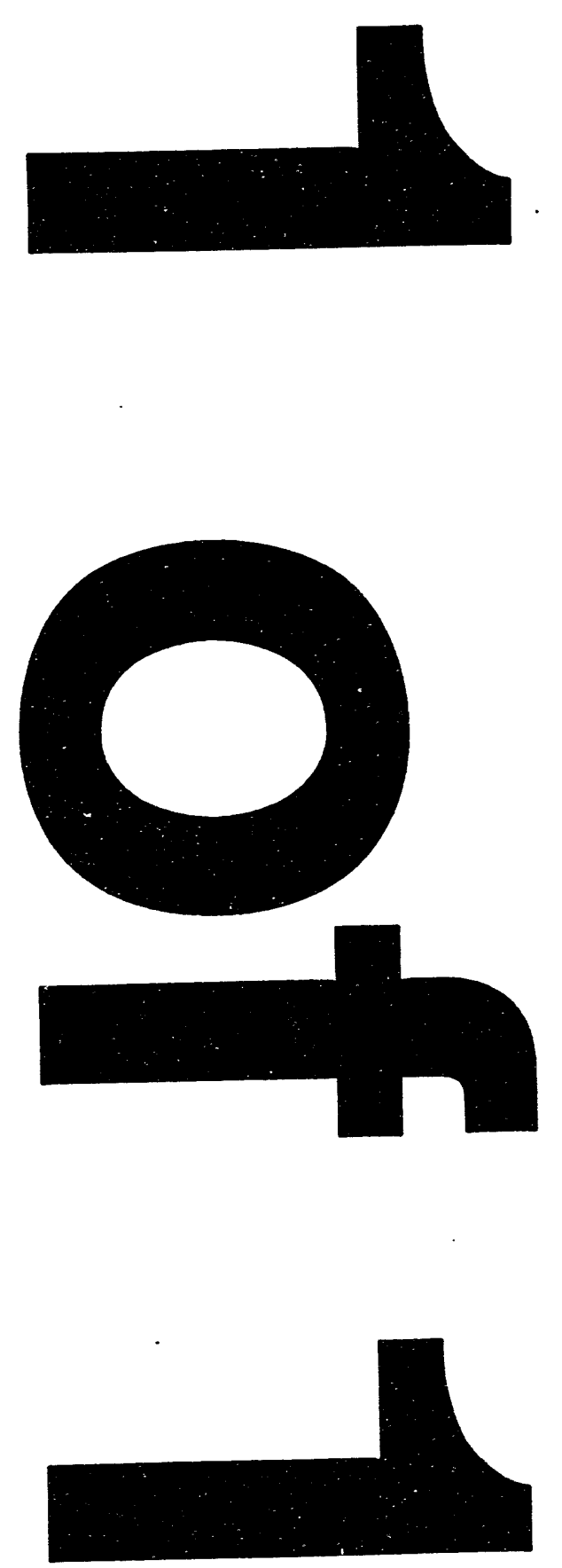
TITLE: THEORY OF METAL INSULATOR TRANSITION IN STRONGLY CORRELATED ELECTRON SYSTEMS

\section{DISCLAIMER}

AUTHOR(S): M. Gulacsi

K. S. Bedell
This report was prepared as an account of work sponsored by an agency of the United States Government. Neither the United States Government nor any agency thereof, nor any of their employees, makes any warranty, express or implied, or assumes any legal liability or responsibility for the accuracy, completeness, or usefulness of any information, apparatus, product, or process disclosed, or represents that its use would not infringe privately owned rights. Reference herein to any specific commercial product, process, or service by trade name, trademark, manufacturer, or otherwise does not necessarily constitute or imply its endorsement, recommendation, or favoring by the United States Government or any agency thereof. The views and opinions of authors expressed herein do not necessarily state or reflect those of the United States Government or any agency thereof.

SUBMITED TO:

Integrated Ferroelectrics Conference Proceedings, International Symposium on Integrated Ferroelectrics, Monterey, CA; March 1994

By acceptance of this article, the publisher reconized that the U S Government retains a nonexclusive, royalty-free license to publish or reproduce the published form of this contribution or to allow others to do so for U S Government purposes.
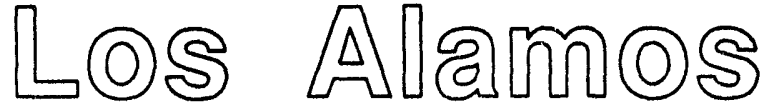

FORM NO. 836 R4 ST. NO. 2829 5/81

\section{Los Alamos National Laboratory Los Alamos, New Mexico 87545}

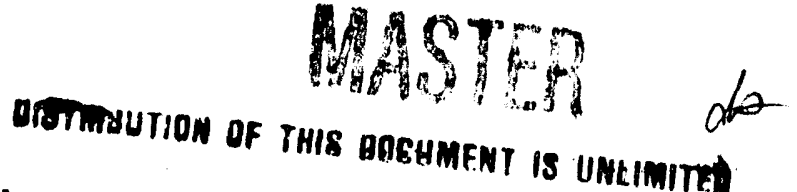




\title{
THEORY OF METAL INSULATOR TRANSITION \\ IN STRONGLY CORRELATED ELECTRON SYSTEMS
}

\author{
M. GULACSI AND K. S. BEDELL \\ Theoretical Division, Los Alamos National Laboratory, \\ Los Alamos, NM 87545, USA.
}

\begin{abstract}
A comprehensive theory of the correlation driven metal insulator transition in 1D and 2D strongly correlated electron systems is given. In both the 1D and 2D Hubbard model the metal insulator transition encountered close to half filling is of Pokrovsky-Talapov type. An important consequence of this in the 2D Hubbard model is the break down of the Fermi liquid theory. We also describe in detail the properties of the Pokrovsky-Talapov transition in 2D ferroelectrics.
\end{abstract}

\section{INTRODUCTION}

One of the most challenging problems in electronic systems is the correlation driven transition from metallic to insulating behavior. The correlation driven metal insulator transition where a disordered metal (a liquid phase) undergoes a transition to an insulating disordered phase as some parameters are varied, is a Mott transition ${ }^{1}$. Thus, the Mott transition occurs without the development of long range order and is typical for strongly correlated electron systems. We consider as a starting point the simplest model in case of which the normal metal is strongly correlated, namely the Hubbard model. In this model the insulating phase sets in because the correlation energy is large enough to prevent the electrons from moving, as opposed to any localization effect driven by randomness. In the following, we will show that the metal insulator transition existing in the 1D and 2D Hubbard model belongs to the the Gruber-Mullins ${ }^{2}$ better known as the Pokrovsky-Talapov ${ }^{3}$ universality class. 


\section{THE POKROVSKY-TA' APOV TRANSITION}

In the past twenty years, incommensurate periodicities have been observed in many physical systems and have become the subject of considerable theoretical interest. Two incommensurate structures in contact can overcome their rigidity and form a common periodicity. This transition is known as the commensurateincommensurate, or lock-in transition.

Since its discovery in the context with dislocation theory ${ }^{4}$, the commensurate-incommensurate transition had a major role ${ }^{5}$ in understanding the physics of charge-density-waves, incommensurate lattice problems, the general problem of incommensurate periodicities, two-dimensional (XY) models, theory of phase transitions in magnetic systems and $1 D$ quantum systems, and the correlation driven metal insulator (Mott) transition ${ }^{6}$.

In most of the systems and models mentioned above, the problem of characterizing the commensurate-incommensurate transition has been reduced to the solution of the 1D sine-Gordon model. The level of incommensurability is measured by a soliton density, $n_{\text {sol. }}(\mu)$, where $\mu$ is the chemical potential. The commensurate-incommensurate transition happens at $\mu=E_{\text {wol. }}$, where $E_{\text {wol. }}$ is the single soliton energy. For $\mu<E_{\text {sol. }}$ and $n_{\text {sol. }}=0$ the system is locked in a commensurate phase, while for $\mu>E_{\text {sol. }}$, i.e., $n_{\text {sol. }} \neq 0$ the structure is incommensurate.

In the classical limit the commensurate-incommensurate transition occurs with the emergence of a soliton lattice in the soliton gap ${ }^{4,7}$. At low soliton density, the solitons repel each other, with an exponential type interaction ${ }^{7}$, and the transition takes place with a logarithmic behavior $n_{\approx 0 l} \propto \ln ^{-1}\left(\mu-E_{\star 0 l}\right)$, i.e., corresponding to a critical exponent $\bar{\beta}=0$. In the full quantum problem, it is known ${ }^{8}$, that the soliton lattice will melt, giving rise to a Luttinger liquid which emerges at the top or bottom of the soliton gap. In this case the solitons will repel each other much stronger than in the classical limit, with an interaction proportional to $n_{\text {sol. }}^{2}$, in the low soliton density limit. The incommensurability close to the transition behaves ${ }^{3}$ as $n_{\text {sol. }} \propto(\mu-\Delta)^{1 / 2}$, where $\Delta$ is the soliton gap, corresponding to a critical exponent $\bar{\beta}=1 / 2$. This is the Pokrovsky-Talapov exponent. In some applications this transition is characterized with the variation of the thermodynamic potential, $\Omega=E\left(n_{\text {sol. }}\right)-\mu n_{\text {sol. }}$, for which we obtain $\Omega \propto n_{\text {sol. }}^{3}=(\mu-\Delta)^{3 / 2}$. From the experimental point of view, the relevant quantity to observe this transition is the charge susceptibility, $\chi_{\text {sol. }}=\left(\partial \mu / \partial n_{\text {sol. }}\right)^{-1}$, which is divergent at the transition $\chi_{\text {sol. }} \propto 1 / n_{\text {eol. }}$. This civergent behavior implies a divergence of the effective mass of the charge carriers, and accordingly the divergence of the specific heat 
coefficient. Thus, the basic characteristics of the Pokrovsky-Talapov transition are:

$$
n_{\text {sol. }}(\mu) \propto(\mu-\Delta)^{1 / 2}, \Omega(\mu) \propto(\mu-\Delta)^{3 / 2}, \chi_{\text {sol. }}(\mu) \propto(\mu-\Delta)^{-1 / 2}
$$

Or, as a function of the soliton density (incommensurability) are:

$$
\mu\left(n_{\text {sol. }}\right) \propto \Delta+n_{\text {sol. }}^{2}, E\left(n_{\text {sol. }}\right) \propto \Delta n_{\text {sol. }}+n_{\text {sol. }}^{3} / 2, \chi\left(n_{\text {sol. }}\right) \propto 1 / n_{\text {sol. }} .
$$

The Pokrovsky-Talapov transition, however, is typical for all integrable models. Its importance in connection to the 2D XY, Gaussian, Coulomb-Gas models but, most important to the equivalent $9,10,11$ (solid-on-solid) surface models led a to thorough analysis of its properties. Recognizing ${ }^{12}$ the equivalence between the Gaussian models and the direct field six-vertex model (defined below) led to the remarkable result that the six-vertex model in a direct field is an ideal model to describe the commensurate-incommensurate transition. The direct field in the six-vertex model correspond to the chemica: potential in Eqs. (1) and (2), while the polarization of the six-vertex model is equivalent to the soliton density, $n_{\text {sol. }}$.

The six-vertex model is the simplest, exactly solvable model describing surface ferroelectric and antiferroelectric transitions. In general, the vertex models define a statistical mechanics on a (for simplicity) square lattice as follows. On each link there is a degree of freedom taking two values which is represented (traditionally) by an arrow, i.e. $\rightarrow$ and $\leftarrow$. To each vector therefore there corresponds 16 possible configurations. To each of these we associate a Boltzmann weight $\omega_{i}=\exp \left(-\varepsilon_{i} / k_{B} T\right)$. This general model is referred to as the sixteen-vertex model. The exact solution of this general ferroelectric model is not known. If we choose the Boltzmann weights, so that six vertices with two entering and two exiting arrows (or eight vertices including also the cases where four or zero arrows exit) have finite weight, then we define the six- or eight-vertex models. The six-vertex model is exactly solvable via the algebraic Bethe Ansatz, for details see Lieb and $\mathrm{Wu}^{13}$ and Baxter ${ }^{14}$. The exact solution has at most four independent variables (this is because the zero energy can be chosen arbitrary and the ice rule implies $\left.\omega_{5}=\omega_{6}\right)^{*}$. In this situation the model is called the asymmetric six-vertex model, in contrast to the symmetric six-vertex model, where the number of independent variables are two. The symmetric model in the presence of $h$ and $v$ external fields is equivalent to the asymmetric one. The energies of the six possible arrow configurations of the symmetric model are $\varepsilon_{1}=\varepsilon_{2}, \varepsilon_{3}=\varepsilon_{4}$ and $\varepsilon_{5}=\varepsilon_{6}$.

* We use the traditional ordering of the vertices, for details see Ref. 14 . 
The exact free energy obtained with the algebraic Bethe Ansatz ${ }^{13,14}$ exhibits three different analytic forms for the three different phases, i.e., two frozenin ferroelectric phases (with the polarization, $p=1$ ), an antiferroelectric phase $(p=0)$ and a disordered phase (paraelectric, $p=0)$. The transitions to the ferroelectric phases is of first order, while the transition from the paraelectric phase to the antiferroelectric one is of Kosterlitz-Thouless type.

Introducing a vertical field, $v$ ithe symmetric model is still easily solvable with the algebraic Bethe Ansatz. All the above mentioned transitions are second order now, as a function of temperature and Pokrovsky-Talapov as function of the external field. The largest eigenvalue of the transfer matrix is determined from the Bethe Ansatz, $z(p)$ in notation of Ref. 13. The exact free energy is obtained as $-F / k_{B} T=\max z(p)_{-1 \leq p \leq 1}$. All the three transitions are similar in nature, accordingly we describe only the antiferroelectric one, $p \rightarrow 0$. The free energy is determined by $z(p) \propto z^{\prime}(0) p+z^{\prime \prime \prime}(0) p^{3} / 6$ [Eq. (304) of Ref. 13] is identical to Eq. (2). Close to the transition $p \propto\left(v-k_{B} T\left|z^{\prime}(0)\right|\right)^{1 / 2}$ [Eq. (340) of Ref. 13], $\Omega \propto\left(v-k_{B} T\left|z^{\prime}(0)\right|\right)^{3 / 2}$ [Eq. (342) of Ref. 13] and the magnetic susceptibility $\chi \propto\left(v-k_{B} T\left|z^{\prime}(0)\right|\right)^{-1 / 2}$ which is equivalent to Eq. (1). The same result is obtained for the full asymmetric six-vertex model, i.e., with both $h$ and $v$ being present, however, due to the complexity of the resulting equations numerical analysis is required.

As different types of (solid-on-solid) crystal surfaces can be described within the six-vertex model, the remarkable feature of the above obtained Pokrovsky-Talapov transition is that it gives a universal curvature for the smoothly curved surfaces in the vicinity of the roughening (Kosterlitz-Thouless) transition. As a third example, we present a variant of the Pokrovsky-Talapov transition, which appears on surfaces, known as the Gruber-Mullins transition ${ }^{2}$.

We will consider a simple example, were the exponents of the transition can be obtained analitically. Namely, we will present the results for the (100), (110) and (101) faces of a simple cubic crystal. In these particular cases, the vertex model corresponding ${ }^{15}$ to these facets is the five-vertex model. The five-vertex model is defined within the frame of the symmetric six-vertex model, with the Boltzmann weight of one of the vertices being strictly zero, e.g. $\omega_{2}=0$, for details see Ref. 16 . In the five-vertex model, in contrast to the symmetric six-vertex model, the arrowreversal symmetry is broken. The model allows an exact solution via the algebraic Bethe Ansatz ${ }^{16}$ and exhibits three differents phases ${ }^{16}$ : two frozen-in ferroelectric phases $(p=1)$, one antiferroelectric phase $(p=0)$ (similar to the symmetric six- 
vertex model) and a ferrielectric phase $(-1 \leq p \leq 1$, instead of the paraelectric of the symmetric six-vertex model). All the phase transitions are of second order ${ }^{16}$. From all these phases, the ferroelectric one corresponds ${ }^{15,17}$ to the above mentioned facets of a simple cubic crystal. Knowing the free energy of the five-vertex model from the Bethe Ansatz equation ${ }^{16}$, we can determine the Cartesian coordinates, $(X, Z)$, of the corresponding surface from the Wulff construction. Being close to the ferroelectric transition, $p \rightarrow 1$, we obtained ${ }^{17}: 1-p \propto\left(X-X_{0}\right)^{1 / 2}, Z-Z_{0} \propto$ $\left(X-X_{0}\right)^{3 / 2}$ and the average spacing between steps $\propto\left(X-X_{0}\right)^{-1 / 2}$, equations identical to Eq. (1).

As mentioned above, a general characteristic of the (Luttinger) liquid phase preceding the Pokrovsky-Talapov transition is that its quasiparticles strongly repel each other. The basic equation obtained for the ground state energy in Eq. (2) can be interpreted as follows: the linear term in $n_{\text {sol. }}$ sums up the energy contributions of the non-interacting quasiparticles (solitons, holons, surface steps, etc., depending on the model under consideration). The missing quadratic term in $n_{\text {sol. }}$ shows that there is no effective attraction between the quasiparticles (see below) and the $n_{\text {sol. }}^{3}$. term suggests of the presence of strong repulsion. This repulsion can be easily understood from the surface variant ${ }^{18}$, to be given by an entropic and elastic interaction. The entropic repulsion ${ }^{2}$ is due to the effect of the non-crossing condition of the quasiparticles (hard core). The elastic repulsion ${ }^{19}$ is mediated through the lattice as the ground state energy is lowest if the quasiparticles are as far from each other as possible.

The Pokrovsky-Talapov transition is very stable with respect to different types of interactions ${ }^{11}$. Short range interactions, with range much less than the average distance between the quasiparticles contribute to the ground state energy by an order of $n_{\text {sol. }}^{4}$ thus, leaving the Pokrovsky-Talapov transition unaltered. This is also the case for any (attractive or repulsive) nearest-neighbour or nextnearest-neighbour interaction on a lattice. An interaction sufficiently long ranged, i.e. $1 / x^{\lambda}$, with $\lambda<2$, will contribute to the ground state an order of $n_{\text {sol. }}^{3}$, again leaving the transition unchanged. The limit $\lambda=2$, which is the only case where the Pokrovsky-Talapov is destroyed, generates a tricritical point for attractive interaction and the emergence of bound states for the repulsive case.

As previously already mentioned, different integrable models are equivalent to each other*. This can be easily seen for the vertex models, where the

* The equivalence stands between a $(x, y) 2 \mathrm{D}$ classical variable space and an $(1+1)$ quantum space. 
configurations of up and down arrows on a vertical bond of the vertex models are evidently isomorphic to the state of a chain of spin $1 / 2$ particles. In order to prove such an isomorphism, we first have to constract the transfer matrix of the vertices in terms of Pauli spin operators and secondly, find a non-trivial linear Hamiltonian which commutes with the transfer matrix. Such a transformation was done for the first time for a special case of the six-vertex model ${ }^{20}$ and of the eight-vertex model $^{21}$. These calculations were extended for the general six- and eight-vertex model by Barouch ${ }^{22}$, Gaudin ${ }^{23}$ and Baxter ${ }^{14}$.

Presently, a whole chain of equivalences is known between the integrable models. To mention a few: the $1 \mathrm{D}$ quantum spin $1 / 2 \mathrm{XXZ}$ model $\longrightarrow^{30}$ six-vertex model $\longrightarrow{ }^{10} 2 \mathrm{D}$ classical surface roughning $\longrightarrow^{24} 2 \mathrm{D}$ classical isotropic XY model $\longrightarrow{ }^{25,26}$ 1D quantum sine Gordon model $\longrightarrow{ }^{27,28}$ 1D quantum Thirring model $\longrightarrow^{26,29}$ 2D classical Coulomb gas or neutral plasma $\longrightarrow^{30}$ 1D quantum fermion gas model $\longrightarrow{ }^{27}$ 1D quantum sine Gordon. On the other hand, the 1D quantum fermion gas model $\longrightarrow^{31}$ 1D quantum spin $1 / 2$ Tomonaga-Luttinger model, or $\longrightarrow{ }^{32} 1 \mathrm{D}$ quantum back-scattering model. However, the $1 \mathrm{D}$ quantum spin $1 / 2$ $\mathrm{XYZ}$ model $\longrightarrow^{22}$ eight-vertex model $\longrightarrow^{33} 1 \mathrm{D}$ quantum interacting neutral field theory (spinless Tomonaga) model $\longrightarrow^{33} 1 \mathrm{D}$ quantum spinless fermion gas (spinless Luttinger) model.

By the above examples we want to emphasize that:

1. The integrable models have common ground state properties* thus, a PokrovskyTalapov type transition will exist in all related models.

2. The previous point is easy to understand, as the algebraic form of the eigenvalues obtained from the Bethe Ansatz equations are identical. However, the same algebraic form generates the characteristic features for such a transition to exist thus, the Pokrovsky-Talapov transition is characteristic to only integrable models in $1 \mathrm{D}$ quantum field theory and $1 \mathrm{D}, 2 \mathrm{D}$ classical statistical mechanics

Concerning the Hubbard model, Shastry ${ }^{35}$ has demonstrated that indeed there existr a family of transfer matrices of two coupled six-vertex models which commutes with the Hubbard Hamiltonian. Thus, from the analogy to sixvertex models and from renormalization group approaches ${ }^{36}$ it is expected that the

* It should be emphasized that the above mentioned mappings between different models establishes an equivalence between the eigenvalues of the different models. That is, the ground state energies and thus, the thermodynamic properties of the different models can be made equivalent. However, non of these mapping schemes preserves the correlation functions, as emphasized in Ref. 34. 
1D Hubbard model will also exhibit a Pokrovsky-Talapov transition. This will be investigated in detail in the following.

\section{THE HUBBARD MODEL}

In this chapter, we will concentrate mostly on the one-dimensional case, which has the advantage to be exactly solvable. This, firstly represents an essential source of information since there is no similar (exactly soluble) theories in higher dimension. Secondly, the Mott transition must depend only on the variation of the ground state energy as a function of different thermodynamic quantities. We found that the characteristic thermodynamic functions are similar in one and higher dimensions which implies that the characteristics of the Mott transition are independent of dimensionality.

\section{$1 \mathrm{D}$ Case}

From the exact Bethe ansatz solution it has already been established that elementary excitations of the Hubbard Hamiltonian fall into three classes. Namely ${ }^{37}$, the excitations of spinless particles (holons), gapless neutral spin excitations (spinons) and charge excitations connected with charge rearrangement. Considering only the charge excitations, the first type mentioned above differs from the ground state only in the momentum distribution of electrons, and disappears as the band filling approaches one half. The second type of charge excitations are connected to electron pairs occupying the same lattice site. That is, we create, e.g., a state of a doubly occupied site. This state corresponds to a pair of complex wave numbers in the exact Bethe ansatz equations ${ }^{37}$.

The hole (electron) excitations correspond to the antikink (kink) magnon-like ("charge-magnon") states of the ferromagnetic Heisenberg chain, By creating pairs of complex wave numbers, antikink-kink or empty-doubly occupied site pair states are formed. Using the exact Bethe ansatz results we will show, as mentioned previously, that bound kink-an'ikink states can appear, corresponding to a bound magnon state of the ferromagnetic Heisenberg chain. Since these states appear above the Hubbard charge gap, they are particle-hole antibonding states, representing excitons, which we will call, "charge-soliton" states.

The energy of $H$ holons and $S$ pairs of complex wave numbers is ${ }^{37}$ :

$$
\varepsilon\left(k_{1}, \ldots, k_{H+2 S}\right)=E_{0}(L)+S U+\sum_{i=1}^{H+2 S} \epsilon\left(k_{i}\right),
$$

where $E_{0}(L)$ is the ground state energy of $L$ electrons (i.e., the half-filled band) and $\epsilon(k)=2 t \cos k+2 t \int_{0}^{\infty} d \xi \exp (-\xi U / 4 t) J_{1}(\xi) \cos (\xi \sin k) /[\xi \cosh (\xi U / 4 t)]$. The 
momentum is (modulo $2 \pi$ ) $p=-\sum_{i=1}^{H+2 S} p\left(k_{i}\right)$ with $p(k)=k+\int_{0}^{\infty} d \xi \exp (-\xi U / 4 t)$ $J_{0}(\xi) \sin (\xi \sin k) /[\xi \cosh (\xi U / 4 t)]$. As the holon pseudo-momenta are not independent of each other ${ }^{37}$, the holons represent interacting quasiparticles. The above formulas of the excitation energies and momenta implicitly define two bands, namely the holon (lower Hubbard, Tomonaga-Luttinger universality class ${ }^{38}$ ) band and the soliton (upper Hubbard, Luther-Emery ${ }^{30,32,39}$ universality class) band.

Using exact solution techniques ${ }^{40}$ and bosonization ${ }^{41}$ much attention has been paid to the repulsive 1D Hubbard model to describe doped Mott insulators and its associated Luttinger liquid behavior. Less interest was devoted to the mapping onto the Luther-Emery model, and the crossover between these two behaviors. Characterizing correctly the above mentioned crossover will allow us to describe the Mott transition. For this, we bosonize the system ${ }^{42,43}$. However, having two bands (the spin excitations are irrelevant for the present calculation) we have developed a new bosonization technique in the sense that we form separate Dirac fields for the lower and upper Hubbard bands, which we bosonize in the standard way 27,41-43. Differences to the well-known bosonization schemes appear in the calculation of the currents, as only the diagonal currents are conserved and the chiral components of different bands are mixed, for details see Refs. 6,44 . In this way we obtain ${ }^{6}$ the effective Hamiltonian describing the charge degrees of freedom to be $H_{c}^{\prime}+H_{c}^{\prime \prime}$, with

$$
\begin{aligned}
& H_{c}^{\prime}=\int d x \frac{1}{2}\left\{\left[\Pi_{c}^{\prime}(x)\right]^{2}+\left[\partial_{x} \Phi_{c}^{\prime}(x)\right]^{2}\right\} \\
& H_{c}^{\prime \prime}=\int d x \frac{1}{2}\left\{\left[\Pi_{c}^{\prime \prime}(x)\right]^{2}+\left[\partial_{x} \Phi_{c}^{\prime \prime}(x)\right]^{2}\right\}+\frac{m^{4}}{\lambda}\left\{1-\cos \left[\frac{\sqrt{\lambda}}{m} \Phi_{c}^{\prime \prime}(x)\right]\right\} .
\end{aligned}
$$

Where $H_{c}^{\prime}$ describes the massless conduction band, while $H_{c}^{\prime \prime}$ is a massive Thirring (quantum sine-Gordon) model describing the upper Hubbard band, where $\lambda / \mathrm{m}^{2}$ is the coupling constant of the model, $\lambda / m^{2}=8 \pi K_{c}^{\prime \prime}$ and $m^{4} / \lambda=U K_{c}^{\prime \prime} / 2 \pi^{2} \alpha_{2}^{2} v_{F}$. The boson fields are rescaled with the corresponding velocities $u_{c / \rho}^{\prime}, u_{c / \rho}^{\prime \prime}$ and with $K_{c / \bullet}^{\prime}\left(K_{c / \bullet}^{\prime \prime}\right)$, the parameter which determines the long-range decay of all correlation functions. At this stage, we can characterize the two bands and calculate the total single-particle Green-functions.

The holons vanish at the exactly half-filling. From Eq. (3), in the thermodynamic limit with $\delta=H / L \rightarrow 0$, we obtain the holon Fermi point $k_{F}^{H} \equiv$ $2 k_{F} \rightarrow \pi$ and, most important, the holon band vanishes as: $\propto-t^{H} \cos k_{F}^{H}$, where $t^{H} \propto \delta$. For $U \ll 1: t^{H}=4 \pi \delta t^{2} / \Delta_{0}(U / t){ }^{*}$ while for any value of $U, t^{H}=\pi / m^{*}$,

* $\Delta_{0}(U / t)$ is the Hubbard gap at half filling. 
with $m^{*}$ being the effective mass of the holons. Thus, approaching half-filling not only the number of holons decreases, but simultaneously the holon band-width ( $\propto$ $\left.\delta t^{2} / \Delta_{0}(U / t)\right)$ vanishes. This observation is the key in understanding the nature of the metal insulator transition and was completely neglected in previous studies ${ }^{40,41}$.

As the holon band-width vanishes, the lower band-width cutoff is exactly $\Lambda_{1}=t^{H} / 2 u_{c}^{\prime}$ which will determine the coefficient of the Luttinger part $\left(H_{c}^{\prime}\right.$ from Eq. (4) of the Green's function $\propto u_{c}^{\prime} \Lambda_{1}\left[\alpha_{1}^{2} /\left(\alpha_{1}^{2}+\left(x-x^{\prime}\right)^{2}\right)\right]^{(\beta+1) / 2}$, where $\beta=\left(K_{c}^{\prime}+1 / K_{c}^{\prime}-2\right) / 4$. Otherwise, the Green's function has its conventional form ${ }^{40,41}$.

It is important to emphasize that the massless Thirring model corresponding to the holon band is an interacting one, more precisely it is selfinteracting ${ }^{6,43}$. Actually, the massless Thirring model is the only self-interacting model in $1+1$ dimension. Going one step further, it can also be demonstrated that, the Lutiinger model corresponding to the holon band, is the Dirac delta function interacting model solved by Mattis and Lieb ${ }^{38}$. This model, more precisely, the momentum distribution of the spinor components, has an essential singularity at $U=0$ where perturbation theory fails. Namely, for any finite value of $\beta$ the momentum distribution of the spinor components is a constant, $\Gamma[(\beta=1) / 2] /[2 \sqrt{\pi} \Gamma(\beta / 2+1)]$ $=1 / 2$ for $U \rightarrow 0$, characteristic to spinless fermion systems. On the other hand, the free field, $U=0$ or $\beta=0$ formulation of the Fermi fields leads to a momentum distribution of a step function. Already Luttinger ${ }^{38}$ noted that the spectrum of the Luttinger model is that of a non-interacting one, independent of the interaction. But, the spinor components of the Fermi fields are different. As mentioned above, the momentum distribution of the left and right movers is not analytic in the $U \rightarrow 0$ limit.

The upper Hubbard band, as seen from Eq. (5) is characterized by a quantum sine-Gordon equation. The two models are equivalent to each other if we impose ${ }^{6}$ that $\left.i\right)$ the Hubbard charge gap $\Delta_{\delta}(U / t)$ is equal to the sine-Gordon soliton mass $M_{\text {sol }}=8 \mathrm{~m} / \gamma: \Delta_{\delta}(U / t) / u_{c}^{\prime \prime}=M_{\iota d}$, where ${ }^{45}$ the renormalized coupling constant $\gamma=\lambda / \mathrm{m}^{2}\left(1-\lambda / 8 \pi \mathrm{m}^{2}\right)^{-1}$ and $\left.i i\right) U \rightarrow \infty$ limit of the half-filled Hubbard model corresponds to the $\lambda / m^{2}=4 \pi$ non-interacting massive Thirring model.

The upper Hubbard band is only slightly affected by doping, where the charge gap varies with doping, as ${ }^{6}: \Delta_{\delta}(U / t)=\Delta_{0}(U / t)+\delta^{2} \Delta_{1}(U / t)$, with $\Delta_{1}(U / t)=I_{1}(2 \pi t / U) / I_{0}^{2}(2 \pi t / U)$. The Green-function given by $H_{c}^{\prime \prime}$ from Eq. (5) is $\propto \exp \left[-\left(\lambda^{2} / m\right) g\left(x-x^{\prime}\right) /(8 \pi)\right]$, where $g\left(x-x^{\prime}\right)=\Delta_{\delta}(U / t)\left|x-x^{\prime}\right| / v_{F}$. The . Green function can be evaluated using the calculated values of $\lambda$ and $m$, obtaining 
the expected exponential decay $\propto \exp \left[-\left|x-x^{\prime}\right| / \xi_{\delta}(U / t)\right]$. This form defines the correlation length $\xi_{\delta}(U / t)=\xi_{\text {sol. }}(U / t)=u_{c}^{\prime \prime} / \Delta_{\delta}(U / t)$ which in the weak-coupling limit equals the soliton length.

The previously obtained exponential decay is due to the presence of the charge gap. The soliton excitation in Eq. (3) are particle-hole excitations across this gap, refering mainly to charge redistribution into empty and doubly occupied sites, are interacting purely through phase shifts. If we treat this excitations (in a first approximation) as quasiparticles being independent of the holons, we can shown that they may even form bound states. For simplicity, we consider a state with two carriers only. We separate out the center-of-mass motion by introducing the center-of-mass wave vector $K=k_{1}+k_{2}$ and the center-of-mass coordinate $R=\left(x_{1}+x_{2}\right) / 2$, and a relative wave vector $q=\left(k_{1}-k_{2}\right) / 2$, and a relative coordinate $r=x_{1}-x_{2}$. In terms of the center-of-mass, and relative wave vectors, we can express $\varepsilon\left(k_{1}, k_{2}\right)$ of Eq. (3) in the form

$$
\begin{aligned}
& \varepsilon(K, q)=E_{0}(L)+\Delta_{0}(U / t)+4 t[1-\cos (K / 2) \cos q] \\
& -8 t \sum_{n=1}^{\infty} f_{2 n}(U / t)(1-\cos K \cos 2 n q),
\end{aligned}
$$

with momenta $p(K, q)=K-4 \sum_{n=0}^{\infty} g_{2 n+1}(U / t) \sin [(2 n+1)(K / 2)] \cos [(2 n+1) q]$. If we consider a hole to be inserted at quasimomenta $k_{1}$, corresponding to a quantum number $I_{1}$, and an electron inserted at $k_{2}$, corresponding to the quantum number $I_{2}$, then the relation between the quasimomenta, in variables $K$ and $q$, is $K=(2 \pi / L)\left(I_{1}+I_{2}+1\right)-2 \int_{0}^{\infty} d \xi \exp (-\xi U / 4 t) J_{0}(\xi) \sin \{\xi[\sin q][\cos (K / 2)]\}$ $\cos \{\xi[\cos q][\sin (K / 2)]\} /[\xi \cosh (\xi U / 4 t)$. Eqs. (6) has been derived from Eq. (3) using the addition theorem of the Bessel functions and the quasimomenta $k_{i}$ were shifted so that the minimum of $\varepsilon\left(k_{i}\right)$ is at $k_{i}=0$. The functions $f_{2 n}(U / t)$ and $g_{2 n+1}(U / t)$ are: $f_{2 n}(U / t)=\int_{0}^{\infty} d \xi \exp (-\xi U / 4 t) J_{1}(\xi) J_{2 n}(\xi) /[\xi \cosh (\xi U / 4 t)$ and $g_{2 n+1}(U / t)=\int_{0}^{\infty} d \xi \exp (-\xi U / 4 t) J_{0}(\xi) J_{2 n+1}(\xi)[\xi \cosh (\xi U / 4 t)$, respectively.

The two carrier eigenstate is a linear combination of all possible configurations of the two charge states, with amplitudes $A_{i, j}$ :

$$
\begin{aligned}
& {\left[E(U)-E_{0}(L)-\Delta_{0}(U / t)-4 t\right.} \\
& \left.+8 t \sum_{n=1}^{\infty} f_{2 n}(U / t)\right] A_{i, j}+t\left(A_{i-1, j}+A_{i+1, j}+A_{i, j-1}+A_{i, j+1}\right) \\
& -2 t \sum_{n=1}^{\infty} f_{2 n}(U / t)\left(A_{i-2 n, j}+A_{i+2 n, j}+A_{i, j-2 n}+\dot{A}_{i, j+2 n}\right)=0 .
\end{aligned}
$$

Subtracting from Eq. (7) the terms orthogonal to it, we obtain the bound state 
equation:

$$
\begin{aligned}
& 1 / 2 t\left[1-2 \sum_{n=1}^{\infty} f_{2 n}(U / t)\right]=\frac{2}{\pi} \int_{0}^{\pi} d q \cos [\cos (K / 2)-\cos q] \\
& \times\left\{E(U)-E_{0}(L)-\Delta_{0}(U / t)-4 t\left[1-2 \sum_{n=1}^{\infty} f_{2 n}(U / t)\right]+4 t \cos (K / 2) \cos q\right. \\
& \left.-8 t \sum_{n=1}^{\infty} f_{2 n}(U / t) \cos K \cos 2 n q\right\}^{-1}
\end{aligned}
$$

Eq. (8) always gives a bound state below the minimum of the continuum $(q=0)$ except for $K=0$, when $E(U)=E_{0}(L)+\Delta_{0}(U / t)$. The quasiparticles forming these states, Eq. (8), are separated from each other as they are antibonding states. Eq. (8) simplifies considerably in the $U=\infty$ limit, where a formula analogous to the bound magnon states of the ferromagnetic Heises lerg model is obtained: $E(\infty)=E_{0}(L)+\Delta_{0}(\infty)+2 t \sin ^{2}\left(K / 2_{i}\right)$.

Finally, the total single-particle Green-function of the Hubbard chain $\left(H_{c}^{\prime}+H_{c}^{\prime \prime}\right)$ is:

$$
\begin{aligned}
& G_{\delta}\left(x-x^{\prime}\right) \propto \delta\left\{\left(\alpha_{1}^{2}\right) /\left[\alpha_{1}^{2}+\left(x-x^{\prime}\right)^{2}\right]\right\}^{(\beta+1) / 2} \\
& +\exp \left[-\left(\left|x-x^{\prime}\right|\right) / \xi_{\delta}(U / t)\right]
\end{aligned}
$$

Eq. (9) is a remarkable result by which, in a natural way and without any approximation we can connect the Tomonaga-Luttinger and Luther-Emery type behavior of the Hubbard model.

To characterize the metal insulator transition we calculate the thermodynamic quantities corresponding to Eqs. (1) and (2). The chemical potential is determined from the momentum distribution. Measuring the energy relative to the bottom of the Hubbard gap $\left[-\Delta_{\delta}(U / t) / 2\right]$ with the notation $\varepsilon_{F}=v_{F} k_{F}$ evaluated at half-filling, we obtain:

$$
\begin{aligned}
& \delta\left(\mu_{-}\right)=\left[2 / \Delta_{1}(U / t)\right]^{1 / 2}\left(\varepsilon_{F}-\mu_{-}\right)^{1 / 2} \\
& \Omega\left(\mu_{-}\right)=\left[\Delta_{1}(U / t) / 3\right]\left[2 / \Delta_{1}(U / t)\right]^{3 / 2}\left(\varepsilon_{F}-\mu_{-}\right)^{3 / 2} \\
& \chi\left(\mu_{-}\right) \propto\left(\varepsilon_{F}-\mu_{-}\right)^{-1 / 2}
\end{aligned}
$$

corresponding to Eq. (1), or

$$
\begin{aligned}
& \mu_{-}(\delta)=\varepsilon_{F}-\delta^{2} \Delta_{1}(U / t) / 2, \\
& E(\delta)=\delta \varepsilon_{F}-\delta^{3} \Delta_{1}(U / t) / 6, \chi(\delta) \propto 1 / \delta,
\end{aligned}
$$

corresponding to Eq. (2). Note the change in sign compared to Eqs. (1) and (2) which is due to the fact that we doped the half filled Hubbard model with holes, 
instead of electrons. The ground state energy from Eq. (11) was already obtained from the Bethe Ansatz in the $U \rightarrow \infty$ limit in Ref. 46.

Eqs. (10) and (11) prove that the Mott transition in the 1D Hubbard model is a Pokrovsky-Talapov transition. Thus, all the characteristics of the Pokrovsky-Talapov transition outlined in the previous chapter are valid for the $1 D$ Hubbard model. Namely, the holons are strongly interacting with an effective repulsive interaction and is no attraction present. The repulsive interaction is mainly due to the non-crossing effect, i.e., hard core. This strong interaction is manifested in the holon effective mass which is obtained from the first derivative of the single particle (holon) energy spectrum for small $\delta: \approx t^{H}=\pi / m^{*}$, where $t^{H}$ is the effective holon hoping element, defined above. In this way we obtain for the effective mass: $m^{*} \propto m_{\text {col. }}^{*} / \delta$, i.e., the effective mass diverges at the transition.

2D Case

For the 2D Hubbard model the only available exact solutions are the Lieb ${ }^{47}$ proof that in the thermodynamic limit the ground state is a singlet, and the Nagaoka theorem ${ }^{48}$. However, the Nagaoka limit is so pathological that the exact solution of two holes ${ }^{49}$ in the $U=\infty$ limit shows that the ground state is already a singlet. Thus, a major understanding regarding the thermodynamic behavior of the 2D Hubbard model is achieved by studying the numerical (Monte Carlo and exact diagonalization) results ${ }^{50}$. These results shows ${ }^{50}$, that close to the transition the chemical potential of the 2D Hubbard model behaves like $\mu(\delta)-\mu_{0} \propto \delta^{2}$, where $\mu_{0}$ is the chemical potential at $\delta=0$. This finding is an important result, as it shows that all the properties enumerated in Eqs. (10) and (11) are valid also in the two-dimensional case. Thus, in the 2D Hubbard model the Mott transition is also a Pokrovsky-Talapov transition.

The above statement is a very powerful one. As it was demonstrated in the previous chapter, the Pokrovsky-Talapov transition is a characteristic of integrable models. Thus, from the above results it it would appear that close to the metal insulator transition the 2D Hubbard model behaves as a one-dimensional quantum system.

In one-dimension, Fermi liquid theory breaks down. The effective low energy theory explicitly exhibits separate elementary excitations carrying charge and spin. The non-Fermi liquid behavior is the basic physics of Eqs. (10) and (11). A Fermi liquid theory ${ }^{51}$ for a dilute Fermi gas will always generate in leading order a form for the chemical potential of $\mu(\delta)-\mu_{0} \propto \mathrm{C}_{0} \delta^{2 / 3}+\mathrm{C}_{1} \delta+\ldots$ in three dimension and $\mu(\delta)-\mu_{0} \propto \mathrm{C}_{1} \delta\left[1+2 / \ln (1 / \delta)+1.23 / \ln ^{2}(1 / \delta)\right]+\ldots$ in two dimension. However, close to the Mott transition the 2D Hubbard moael behaves as in Eq. (11) which 
shows the break down of the Fermi liquid theory. Considering the fact that the Pokrovsky-Talapov transition is characteristic to 1D quantum systems, this result suggest that some sort of Luttinger type behavior is possible. At present, work is in preparation to elucidate this question.

A further important consequence of Eqs. (10) and (11) is the existence of strong repulsive interaction between the quasiparticles, due to non-crossing, hard core effect. This behavior generates a diverging effective mass close to the Mott transition, consistent with the numerical findings ${ }^{50}$. Any attractive interaction will generate a condensate (spin-density-wave, charge-density-wave or superconductivity), i.e. an order parameter $\Delta$ which contributes to the ground state as $\propto \Delta^{2} \approx \delta^{2}$. The absence of the quadratic term in the expansion of the ground state energy signals the absence of any attractive force, in two- and three-dimensions. The one-dimensional systems are special because even the transition to the band insulator has the same singularity. In $1 \mathrm{D}^{7}$ the dispersion in the presence of a gap in the electronic spectrum is of the form $E(k) \propto\left(\Delta^{2}+k^{2}\right)^{1 / 2}$ type. Thus, the soliton density given by the difference in the density of added and removed electrons: $\left.\left.n_{\text {sol. }}=\int_{-\infty}^{\infty}(d k / 2 \pi)\left\{\left[\exp (E(k)+\mu) / k_{B} T\right]+1\right]^{-1}-\left[\exp (E(k)-\mu) / k_{B} T\right]+1\right]^{-1}\right\}$, which at $T=0$ becomes $n_{\text {sol. }}=\left(\mu^{2}-\Delta^{2}\right)^{1 / 2} \operatorname{sign} \mu \theta(|\mu|-\Delta) / \pi$. In first approximation $\mu \approx \Delta$ and the energy becomes $E\left(n_{\text {sol. }}\right)=\Delta n_{\text {sol. }}\left[1+\pi^{2} n_{\text {wol. }}^{2} / 6 \Delta^{2}\right]$. However, this is not the case for dimensions higher than one.

\section{REFERENCES}

1. N. F. Mott, Metal-Insulator Transition, Taylor \& Francis, 1974.

2. E. E. Gruber and W. W. Mullins, J. Phys. Chem. Solids, 28, 875 (1967).

3. V. L. Pokrovsky and A. L. Talapov, Sov. Phys. JETP, $\underline{48}, 579$ (1978); Phys. Rev. Lett., 42, 65 (1979); and Sov. Phys. JETP, 51, 134 (1980).

4. F. C. Frank and J. H. van der Merwe, Proc. Royal Soc., A198, 205 (1949).

5. For a review, see P. Bak, Rep. Prog. Phys., 45, 587 (1982).

6. M. Gulacsi and K. S. Bedell, Phys. Rev. Lett., 72, 2765 (1994).

7. B. Horowitz, J. Phys., C1ㅗ, 161 (1982).

8. F. D. M. Haldane, J. Phys., A 15,507 (1982).

9. S. T. Chui and J. D. Weeks, Phys. Rev., B14, 4978 (1976).

10. H. van Beijeren, Phys. Rev. Lett., 38, 993 (1977); and C. Jayaprakash and W. F. Saam, Phys. Rev., B30, 3917 (1984).

11. C. Jayaprakash, C. Rottmenn and W. F. Saam, Phys. Rev., B 30, 6549 (1984).

12. M. P. M. den Nijs, Phys. Rev., B23, 6111 (1981); and B. Horowitz, T. Bohr, 
J. M. Kosterlitz and H. J. Schulz, Phys. Rev., B 28,6596 (1983).

13. E. H. Lieb and F. Y. Wu, in Phase Transition and critical Phenomena, edited by C. Domb and N. S. Green, vol. 1, Academic Press, 1972.

14. R. J. Baxter, Exactly Solvable Models in Statistical Mechanics, Academic Press, 1990.

15. C. Garrod, A. C. Levi and M. Touzani, Solid State Commun., 75 , 375 (1990).

16. M. Gulacsi, H. van Beijeren and A. C. Levi, Phys. Rev., E447, 2473 (1993).

17. M. Gulacsi, A. C. Levi and E. Tosatti, Phys. Rev., E, in press.

18. H. van Beijeren and I. Nolden, in Structure and Dynamics of Surfaces II, ed. by W. Schommers and P. von Blanckenhagen, Topics in Current Physics, Springer Series, 1987, p. 259.

19. J. Villain, D. R. Grempel and J. Lapujoulade, J. Phys., F15, 809 (1985).

20. B. McCoy and T. T. Wu, II Nuovo Cimento, BLVI, 311 (1968).

21. B. Sutherland, J. Math. Phys., 11, 3183 (1970).

22. E. Barouch, in Ref. 13.

23. M. Gaudin, La fondation d'onde de Bethe, Masson, 1983.

24. J. V. Jose, L. P. Kadanoff, S. Kirkpatrick and D. R. Nelson, Phys. Rev., B1ㅡ, 1217 (1977).

25. J. V. Jose, Phys. Rev., D14, 2826 (1975).

26. J. Zittarz, Z. Physik, B $\underline{23}, 55$ (1976).

27. R. Heindenreich, B. Schroer, R. Seiler and U. Uhlenbrock Phys. Lett, A $\underline{54}, 119$ (1975).

28. S. Coleman, Phys. Rev., D 11,2088 (1975); and S. Mandelstam, Phys. Rev., D11, 3026 (1975).

29. J. M. Kosterlitz and D. J. Thouless, J. Phys., C 6 , 1181 (1973).

30. P. A. Lee, Phys. Rev. Lett., 34, 1247 (1975).

31. A. Luther, Phys. Rev., B 14,2153 (1976); and ibid., B 15,403 (1977).

32. A. Luther and V. J. Emery, Phys. Rev. Lett., 33, 589 (1974).

33. A. Luther and I. Peschel, Phys. Rev., B 11,205 (1975).

34. J. D. Johnson, S. Krinsky and B. M. McCoy, Phys. Rev., Aㅁ, 2526 (1973).

35. B. S. Shastry, Phys. Rev. Lett., $\underline{56}, 1529,2453$ (1986); and J. Stat. Phys., $\underline{50}$, 57 (1988).

36. T. Giamarchi, Phys. Rev., B $\underline{44}, 2905$ (1991); E. B. Kolomeisky, Phys. Rev., B47, 6193 (1993); and J. P. Straley and E. B. Kolomeisky, Phys. Rev., B $\underline{48}, 1378$ (1993).

37. A. A. Ovchinnikov, Sov. Phys. JETP, 30,1160 (1970); I. A. Muurkin and A. 
A. Ovchinikov, Sov. Phys. Solid State, 12, 2031 (1971); C. F. Coll, III, Phys. Rev., B $\underline{9}, 2150$ (1974); F. Woynarovich, J. Phys., C1ㅗ, 85, 97 (1982), C1ㅡ, 5293 (1983); and N. Kawakami and A. Okiji, Phys. Rev., B $\underline{40}, 7066$ (1989).

38. S. Tomonaga, Prog. Theor. Phys., $\underline{5}, 544$ (1950); J. M. Luttinger, J. Math. Phys., 4,1154 (1963); D. C. Mattis and E. H. Lieb, J. Math. Phys., 6, 304 (1965); and I. Ye. Dzyaloshinskii and A. I. Larkin, Sov. Phys. JETP, 38, 202 (1973).

39. V. J. Emery, A. Luther and I. Peschel, Phys. Rev., B $\underline{13}, 1272$ (1976); and A. Luther, Phys. Rev., A14, 2153 (1976).

40. R. Heidenreich, R. Seiler and D. A. Uhlenbrock, J. Stat. Phys., 22, 27 (1980); and N. Kawakami and S. -K. Yang, J. Phys. Cond. Matter, 3 , 5983 (1991).

41. H. J. Schulz, Phys. Rev. Lett., $\underline{64}, 2831$ (1990); and Int. J. Mod. Phys., B $\underline{5}$, 57 (1991).

42. V. J. Emery, in Highly Conducting One-Dimensional Solids, edited by J. T. Devereese, et al., Plenum, 1979, p. 327; F. D. M. Haldane, Phys. Rev. Lett., $\underline{45}$, 1358 (1980) and ibid., 47, 1840 (1981); T. Giamarchi and H. J. Schulz, Phys. Rev., B $\underline{39}, 4620$ (1989); and E. Fradkin, Field Theories of Condensed Matter Systems, Addison-Wesley, 1991.

43. Y. K. Ha, Phys. Rev., D $\underline{29}, 1744$ (1984).

44. M. Gulacsi and K. S. Bedell, J. Phys. Chem. Solids, 54 , 1149 (1993).

45. R. Rajaraman, Solitons and Instantons, Noth-Holland, 1989 and the references cited therein.

46. H. Shiba, Phys. Rev., B $\underline{6}, 960$ (1972); and E. Tosatti and L. Yu, Physica, C1 153 $-\underline{155}, 1253$ (1988).

47. E. H. Lieb, Phys. Rev. Lett., 62, 1201 (1989).

48. Y. Nagaoka, Phys. Rev., 147, 392 (1966).

49. E. V. Kuz'min, Sov. Phys. JETP Lett., 57,591 (1993).

50. N. Furukawa and M. Imada, J. Phys. Soc. Japan, 60, 3604 (1991); $\underline{61}, 3331$ (1992); 62, 2557 (1993); and Physica, B $\underline{186}$ - $\underline{188}, 931$ (1993).

51. L. D. Landau and E. M. Lifshitz, Statistical Physics, Part. 2, Pergamon Press, 1982. 

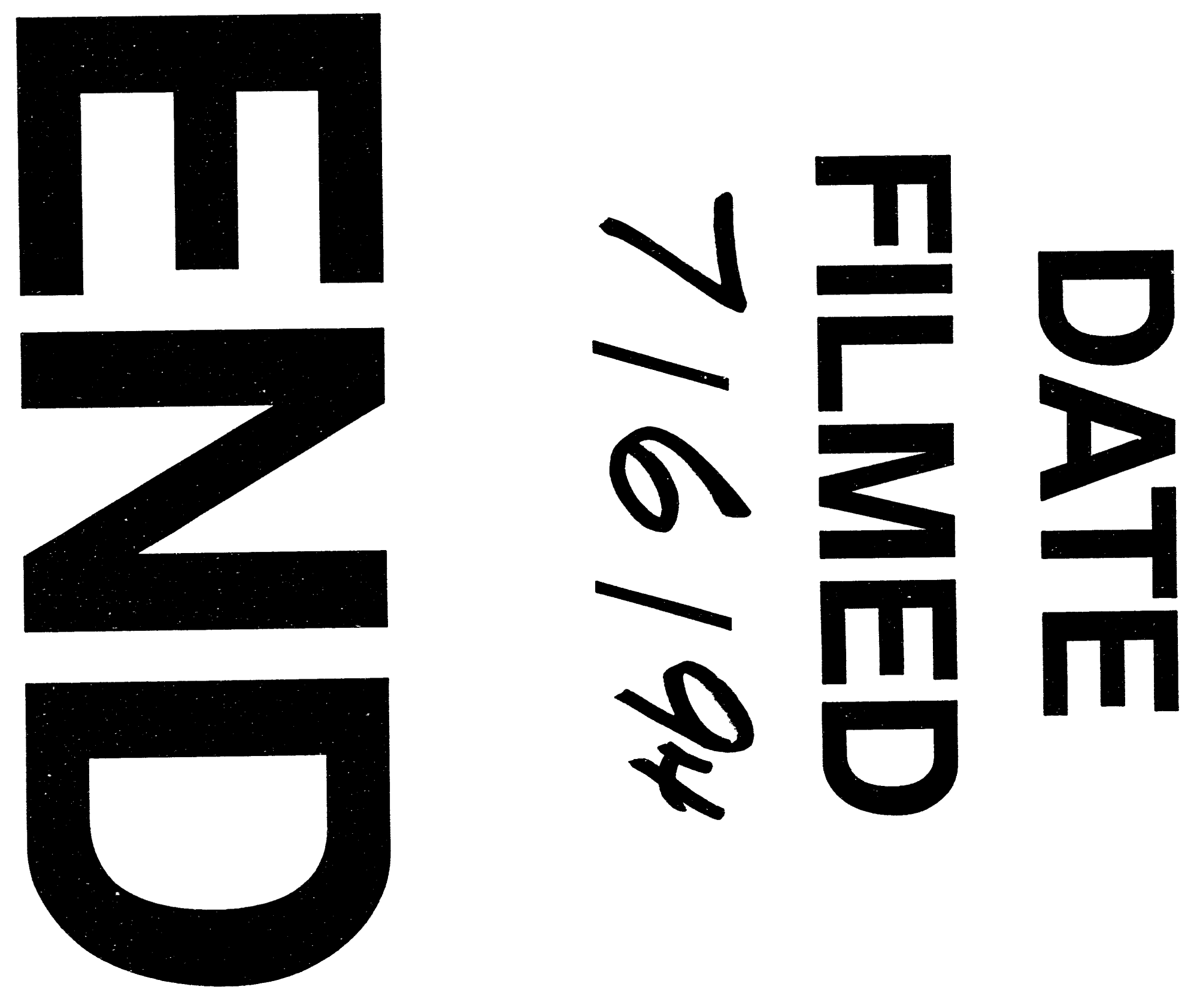

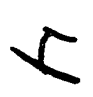




\section{L.}

